\title{
Serotonins of safflower seeds play a key role in anti-inflammatory effect in lipopolysaccharide-stimulated RAW 264.7 macrophages
}

\author{
Dong-Hee Kim $\cdot$ Yong-Sun Moon $\cdot$ Tae-Soon Park $\cdot$ Jun-Ho Son
}

Received: 6 October 2015 / Revised: 2 December 2015 / Accepted: 2 December 2015

(c) Korean Society for Plant Biotechnology

\begin{abstract}
Safflower (Carthamus tinctorius) seeds are wellknown traditional oriental medicines that have long been used for the remedies of blood stasis and bone formation in east Asia. In this study, ethyl acetate (EtOAc) was used for extraction of the main chemical compounds from C. tinctorius seeds. Four major compounds were identified, acacetin, cosmosiin, $\mathrm{N}$-feruloyl serotonin and $\mathrm{N}$-( $p$-coumaroyl) serotonin. Each compound was evaluated for its inhibitory activity against the inflammatory process of macrophages. All compounds significantly inhibited production of lipopolysaccharide (LPS)stimulated nitric oxide (NO) and pro-inflammatory cytokines. The protein levels of inducible nitric oxide synthase (iNOS) and cyclooxygenase-2 (COX-2) were dramatically decreased by serotonins in a dose-dependent manner in LPS-stimulated RAW 264.7 macrophages. These results suggest that serotonin derivatives from safflower seeds may reduce inflammationrelated diseases.
\end{abstract}

Keywords Anti-inflammatory, LPS-stimulated RAW 264.7 macrophages, Safflower seeds, Serotonins

\section{Introduction}

The flowers and leaves of safflower (Carthamus tinctorius L.), which belongs to chrysanthemum family, have long been used to treat stroke, gynecological diseases, coronary heart disease, angina pectoris and hypertension in traditional Chinese

\footnotetext{
${ }^{\dagger}$ Two authors equally contributed in this research.

D.-H. Kim ${ }^{\dagger} \cdot$ T.-S. Park · J.-H. Son $(\bowtie)$

Team of Product Development, Daegu Gyeongbuk Institute for Oriental Medicine Industry, Gyeongsan-si, 38540, Republic of Korea e-mail: bio115@dgom.re.kr

Y.-S. Moon ${ }^{\dagger}$

Dept. of Horticulture \& Life Science, Yeungnam University, Gyeongsan-si, 38541, Republic of Korea
}

medicines (Han et al. 2009; Li et al. 2009; Mandade et al. 2012). Additionally, C. tinctorius seeds, which are rich in unsaturated fatty acid and a-linoleic acid, are widely used for the treatment of blood stasis, bone formation, and osteoporosis and prevention of rheumatism in Korea (Kim 1998; Seo et al. 2000)

Inflammation is a defense mechanism against harmful microorganisms, but the persistence of this state is related to many adult diseases. Although inflammatory mediators like nitric oxide (NO), prostaglandin $\mathrm{E}_{2}\left(\mathrm{PGE}_{2}\right)$, interleukins (IL-1 $\beta$, IL-6), and tumor necrosis factors- $\alpha(\mathrm{TNF}-\alpha)$ are crucial in a defense mechanism, over expression of these mediators may provoke chronical diseases (Delgado et al., 2003; Kubes and McCafferty, 2000). Lipopolysaccharide (LPS) treated macrophages are often used to investigate the suppression of inflammation of many different samples. LPS can induce inflammatory mediators in order to imitate the real conditions in RAW 264.7 macrophages (Hewett and Roth 1993; Watson et al. 1999). During LPS-stimulation, inducible NO synthase (iNOS) and cyclooxygenase-2 (COX-2) can produce $\mathrm{NO}$ and $\mathrm{PGE}_{2}$, respectively (Abdelrahman et al. 2005; Makarov 2000). Inhibited expression of iNOS and COX-2 is thoroughly for its potential to prevent inflammatory diseases and cancer (Surh and $\mathrm{Na}$ 2008). Although various biological activities of $C$. tinctorius flowers and leaves have been reported (Sakamura et al. 1978), there have been few investigations of their inhibitory activity with respect to inflammation. Roh et al. report is pretty much the only research paper about a whitening effect using safflower (C. tinctorius L.) seeds (Roh et al. 2004). We previously reported that the partially purified ethyl acetate (EtOAc) soluble fraction of $C$. tinctorius seeds showed inhibition of cytokine, iNOS and COX-2 expression (Kim et al. 2013). Further, we purified and identified four major chemicals from seed extract and explored their anti-inflammatory activity in LPS-stimulated RAW264.7 macrophages in vitro. 


\section{Materials and Methods}

Seed extraction and chemical identification

Safflowers (C. tinctorius $\mathrm{L}$.) were grown by contract farming, and seeds were purchased in 2011 from Humanherb Co. Five kilograms of air-dried $C$. tinctorius seeds were extracted with n-hexane for purification of bioactive compounds using the protocol described by Kim et al. (Kim et al. 2012). After removing the n-hexane layer, the aqueous solution was extracted three times with $70 \%$ ethanol (EtOH) under reflux for 4 hours. The EtOH extract was then filtered and exhaustively concentrated to give an EtOH extract $(615 \mathrm{~g})$. After sequential purification with various solvents, final fractions of n-hexane, EtOAc, and n-BuOH were obtained $15.8 \mathrm{~g}, 288.6 \mathrm{~g}$, and $182.9 \mathrm{~g}$ respectively. The EtOAc fraction (200 g) was then applied to silica gel $(7.5 \times 50 \mathrm{~cm})$ and eluted with $\mathrm{CHCl}_{3}: \mathrm{MeOH}(10$ : 1) to separate into six fractions (Fr.1-6). Fr.1 and Fr. 5 were combined and crystallized from cold methanol to yield (1) acacetin $(7.74 \mathrm{mg}$ ) and (2) cosmosin (4.76 mg). Fr. 4 was subjected to further chromatography on a silica gel column (4 $\mathrm{X} 30 \mathrm{~cm})$ and then eluted with $\mathrm{CHCl}_{3}: \mathrm{MeOH}(5: 1)$ to afford five sub-fractions (Fr.4.1-Fr.4.5), after which (3) N-feruloyl serotonin $(6.13 \mathrm{mg})$ was crystallized from Fr. 4.2. Further chromatography of Fr.4.3 was conducted on a silica gel column $(4 \mathrm{X} 30 \mathrm{~cm})$ and then eluted with $\mathrm{CHCl}_{3}: \mathrm{MeOH}(10$ : 1) to yield (4) $\mathrm{N}$-( $p$-coumaroyl) serotonin $(15.33 \mathrm{mg})$. Analytical TLC was performed on pre-coated silica gel 60 F254 plates or RP-18 F254. For column chromatography, silica gel Kiesel gel 60 was used. All silica gels were purchased from Merck (Merck, Readington, NJ, USA). After re-confirming the chemicals by liquid chromatography (LC), the chemical structures were analyzed by ${ }^{13} \mathrm{C}-\mathrm{NMR}$ spectroscopy using a 500MHz FT-NMR spectrometer (Jeol, ToKyo, Japan).

Cell culture and cell viability

Mouse RAW 264.7 macrophages were purchased from the Korean Cell Line Bank (http://www.atcc.org/). Cells were grown at $37^{\circ} \mathrm{C}$ under a humidified atmosphere $\left(5 \% \mathrm{CO}_{2}\right)$ in Dulbeco's Modified Eagle's Medium (DMEM) (Sigma, St. Louis, MO, USA) containing 10\% fetal bovine serum, 100 $\mathrm{IU} / \mathrm{mL}$ penicillin $\mathrm{G}$, and $100 \mathrm{mg} / \mathrm{L}$ streptomycin (Gibco BRL, Grand Island, NY, USA). Cell viability was measured using a 3-(4,5-dimethylthiazol-2-yl)-2,5-diphenyltetrazolium bromide (MTT) assay. Briefly, cells (3 X 10 in $200 \mu \mathrm{L} /$ medium) were treated with $20 \mu \mathrm{L}$ of each extract and then incubated for 24 hours at $37^{\circ} \mathrm{C}$. MTT reagent $(0.5 \mathrm{mg} / \mathrm{L})$ (Sigma, St. Louis, $\mathrm{MO}$, USA) was then added to the medium, after which the samples were incubated for an additional 4 hours. Following removal of the medium, MTT-formazan was dissolved in $200 \mu \mathrm{L} / \mathrm{mL}$ dimethylsulfoxide (DMSO). The extent of the reduction of MTT to formazan was quantified by measuring the absorbance at $540 \mathrm{~nm}$ using a microplate reader (Sunrise, Tecan, Salzburg, Austria).

Anti-inflammation assay

$\mathrm{NO}$ and $\mathrm{PGE}_{2}$ production were measured for analysis of the inflammatory inhibitory effect of $C$. tinctorius seed extracts. NO liberated from RAW 264.7 macrophages was measured using a Griess assay according to the method described by $\mathrm{Kim}$ et al. (Kim et al. 2013). The medium was subsequently mixed with an equal volume of Griess reagent (Sigma, St. Louis, MO, USA), after which the amount of NO was quantified by measuring the absorbance at $540 \mathrm{~nm}$ using a microplate reader and comparing the values to a sodium nitrite standard curve. The concentration of $\mathrm{PGE}_{2}$ and cytokines in the supernatant of macrophage cultures was determined by enzyme-linked immuno sorbent assay (ELISA) using an antibody from R\&D Systems according to the manufacturer's instructions (R\&D Systems, Minneapolis, MN, USA).

Western blot analysis

After the extracts were prepared at concentrations of 6.25 , 12.5 , or $25 \mu \mathrm{g} / \mathrm{L}$, treated RAW 264.7 macrophages were incubated for 18 hours at $37^{\circ} \mathrm{C}$. The cells were subsequently harvested by washing with PBS, lysed with $100 \mu \mathrm{L}$ of RIPA buffer (pH 7.4) composed of $50 \mathrm{mM}$ Tris, $0.1 \%$ SDS, $50 \mathrm{mM}$ $\mathrm{NaCl}, 1 \% \mathrm{NP}-40,1 \mathrm{mM}$ PMSF, $10 \mu \mathrm{g} / \mathrm{L}$ of aprotinin and leupeptin, and then centrifuged at 12,000 rpm for 20 minutes. The protein content in the supernatant was quantified using a BioRad protein assay kit (BioRad, Hercules, CA, USA). Briefly, $20 \mu \mathrm{g}$ of proteins were analyzed by $10 \%$ SDS-PAGE, then transferred onto PVDF membranes using a Trans-Blot apparatus (BioRad, Hercules, CA, USA). Following transfer, the membrane was blocked with $5 \%$ nonfat dried milk (w/v) in TBS-T for 1 hour at RT. After six washes, the membrane was incubated with primary iNOS and COX-2 rabbit polyclonal antibodies from Santa Cruz Biotechnology (Santa Cruz Biotechnology, Santa Cruz, CA, US), then diluted $(1: 1,000)$ with TBS-T for 12 hours at $4^{\circ} \mathrm{C}$. After a strong wash, samples were incubated with mouse anti-rabbit $\operatorname{IgG} \operatorname{HRP}(1: 1,000)$ for 2 hours at RT. The samples were then washed again, after which proteins were detected using an Enhanced Chemiluminescence kit (Amersham Bioscience, Little Chalfont, UK). The detection reagent was poured onto the membrane and incubated for 1 
minute, after which the band density was quantified with a LAS4000 image analyzer (Fujifilm Life Science, Tokyo, Japan).

Statistical analysis

Analysis of variance was performed using SPSS (SPSS Inc., Chicago, IL, USA). All data are expressed as the means \pm SD. Differences among means were determined by Tukey's range test ( $t$-test) with $\mathrm{p}<0.05$ and $\mathrm{p}<0.01$ considered to indicate significance.

\section{Results and Discussion}

Identification of four major chemical compounds

We previously demonstrated that the EtOAc fraction of Carthamus tinctorius seeds generated the highest inhibitory effect of inflammation among various extraction buffers, including 70\% EtOH, n-hexane, ethyl acetate, and butyl alcohol (Kim et al. 2013). In this study, partially purified

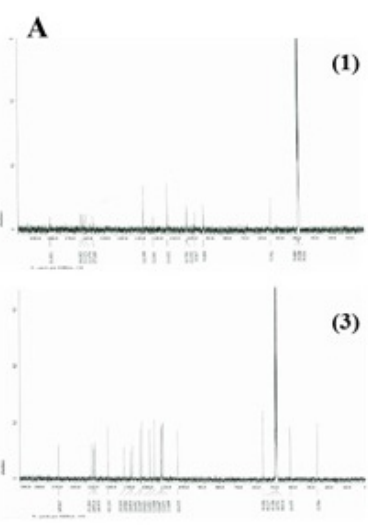

B

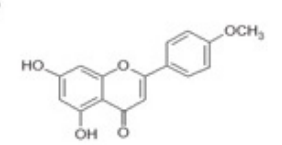

(1)

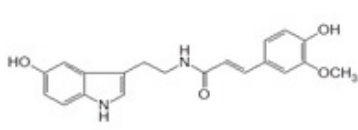

(3)

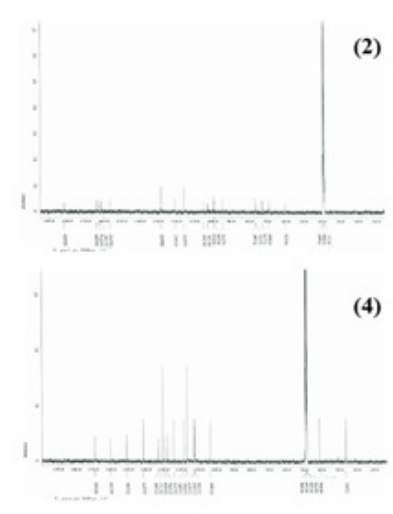

(4)

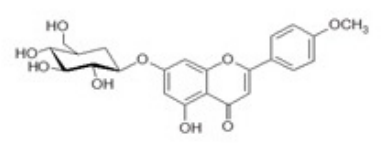

(2)

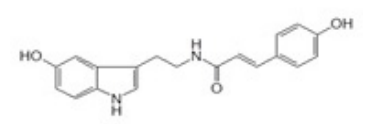

(4)
Fig. 1 Chemical structures of major four compounds from the ethyl acetate extract of $C$. tinctorius seeds. (A) NMR data showing the highest peak from 13C labelled major chemicals (B) chemical structures of (1) acacetin, (2) cosmosiin, (3) N-feruloyl serotonin and (4) N-(p-coumaroyl) serotonin
EtOAc seed extract was further fractionated and major peaks were isolated using a modified method described by Kim et al. (2012). The four major compounds were identified as acacetin, cosmosiin, $\mathrm{N}$-feruloyl serotonin and $\mathrm{N}$-( $p$-coumaroyl) serotonin by ${ }^{13} \mathrm{C}$ labelled NMR (Fig. 1A and 1B). The main peaks and chemical structures were similar to those identified in former studies (Sakamuta et al. 1980).

$\mathrm{NO}$ and $\mathrm{PGE}_{2}$ levels were decreased by treatment with each major compound

When animals get infected, inflammation is started as the first reaction of the immune system by cytokines, $\mathrm{NO}$ and $\mathrm{PGE}_{2}$. Kim et al. (2013) reported that crude extract of C. tinctorius seeds decreased inflammation in macrophages (Kim et al. 2013). In this study, four compounds (acacetin, cosmosiin, $\mathrm{N}$-feruloyl serotonin and $\mathrm{N}$-(p-coumaroyl) serotonin) were purified and identified, after which each compound was evaluated for its ability to modulate the regulatory mechanism of NO, cytokines, $\mathrm{PGE}_{2}$, iNOS and COX-2 in LPS-activated RAW264.7 cells. To investigate key anti-inflammatory chemicals among major compounds, a colorimetric MTT assay was used for cell toxicity. The results showed that the four compounds had little or no effect on cell viability (data not shown). LPS was added in mouse RAW 264.7 macrophages to produce cytokines such as TNF- $\alpha$, IL-1 $\beta$, and IL-6. The levels of pro-inflammatory cytokines were significantly reduced by all compounds (1) - (4). N-feruloyl serotonin (Fig. 1C) showed the most inhibitory effect of TNF- $\alpha$, IL-1 $\beta$, and IL-6, with $\mathrm{IC}_{50}$ values of $20.7 \pm 1.62,12.8 \pm 2.15$ and $11.1 \pm 0.44 \mu \mathrm{M}(\mathrm{p}<0.05$, $\mathrm{p}<0.001)$, when compared with pyrrolidine dithiocarbamate (PDTC) as a positive control $\left(\mathrm{IC}_{50}=27.3 \pm 0.51,21.7 \pm 2.17\right.$ and

Table 1 Effect of Four Major Compounds from Ethyl Acetate Extract of $C$. tinctorius Seeds on TNF-a, IL-1 $\beta$, and IL-6 Production in RAW 264.7 Macrophages

\begin{tabular}{cccc}
\hline \multirow{2}{*}{ Compound } & \multicolumn{3}{c}{$\mathrm{IC}_{50}(\mu \mathrm{M})^{\mathrm{Z}}$} \\
\cline { 2 - 4 } & $\mathrm{TNF}-\alpha$ & $\mathrm{IL}-1 \beta$ & $\mathrm{IL}-6$ \\
\hline Acacetin & $35.2 \pm 3.11$ & $19.6 \pm 2.51$ & $21.1 \pm 6.14$ \\
Cosmosiin & $32.4 \pm 0.54$ & $18.4 \pm 0.48$ & $17.1 \pm 1.81$ \\
$N$-feruloyl serotonin & $20.7 \pm 1.62$ & $12.8 \pm 2.15$ & $11.1 \pm 0.44$ \\
$N$-(p-coumaroyl) serotonin & $27.3 \pm 0.84$ & $16.8 \pm 3.16$ & $17.7 \pm 2.14$ \\
PDTC & $23.3 \pm 0.51$ & $21.7 \pm 2.17$ & $21.1 \pm 1.69$ \\
\hline
\end{tabular}

${ }_{\mathrm{Z}} \mathrm{IC}_{50}$ values were determined by regression analysis and expressed as the mean $\pm \mathrm{SD}$ of three separate experiments. PDTC was used as a positive control. Production of TNF- $\alpha$, IL-1 $\beta$, and IL-6 in various concentrations of major compounds was measured, and the $50 \%$ of inhibition concentration $\left(\mathrm{IC}_{50}\right)$ was calculated by the formula. $\mathrm{IC}_{50}=[\mathrm{I}] /\left[\mathrm{v}_{\mathrm{o}} / \mathrm{v}_{\mathrm{i}}-1\right]$, where vo is the control reaction rate without inhibitor and vi is the rate with inhibitor at concentration [I]. 
21.1 $\pm 1.69 \mu \mathrm{M})$ (Table 1). The two serotonin derivatives (Fig. $1 \mathrm{C}$ and 1D) highly suppressed expression of inflammatory mediators especially cytokines when compared to other flavonoids (Fig. 1A and 1B). From the results, the location of the feruloyl group may influence inhibition of pro-inflammatory cytokines. Especially the two serotonin derivatives, $\mathrm{N}$-feruloyl serotonin and $\mathrm{N}$-(p-coumaroyl) serotonin, significantly suppressed the production of pro-inflammatory cytokines.

NO, a crucial secondary messenger, is synthesized from L-arginine (Palmer et al. 1988). In the inflammatory mechanism, $\mathrm{NO}$ and $\mathrm{PGE}_{2}$ are produced by iNOS (Xie and Nathan 1994) and COX-2, respectively (Tannenbaum et al. 1996). Inhibitions of $\mathrm{NO}$ and $\mathrm{PGE}_{2}$ production were also investigated in LPSstimulated macrophages. In Figure 2, PDTC at $25 \mu \mathrm{M}$ inhibited $\mathrm{NO}$ and $\mathrm{PGE}_{2}$ by $54 \%, 60 \%, 55 \%$ and $75 \%$, respectively. $\mathrm{NO}$ and $\mathrm{PGE}_{2}$ levels were significantly decreased by the compounds, whereas only LPS was treated as a negative control caused increased $\mathrm{NO}$ and $\mathrm{PGE}_{2}$ production (Fig. 2). Therefore, we investigated more on the relationship between the compounds and iNOS and COX-2 expression.
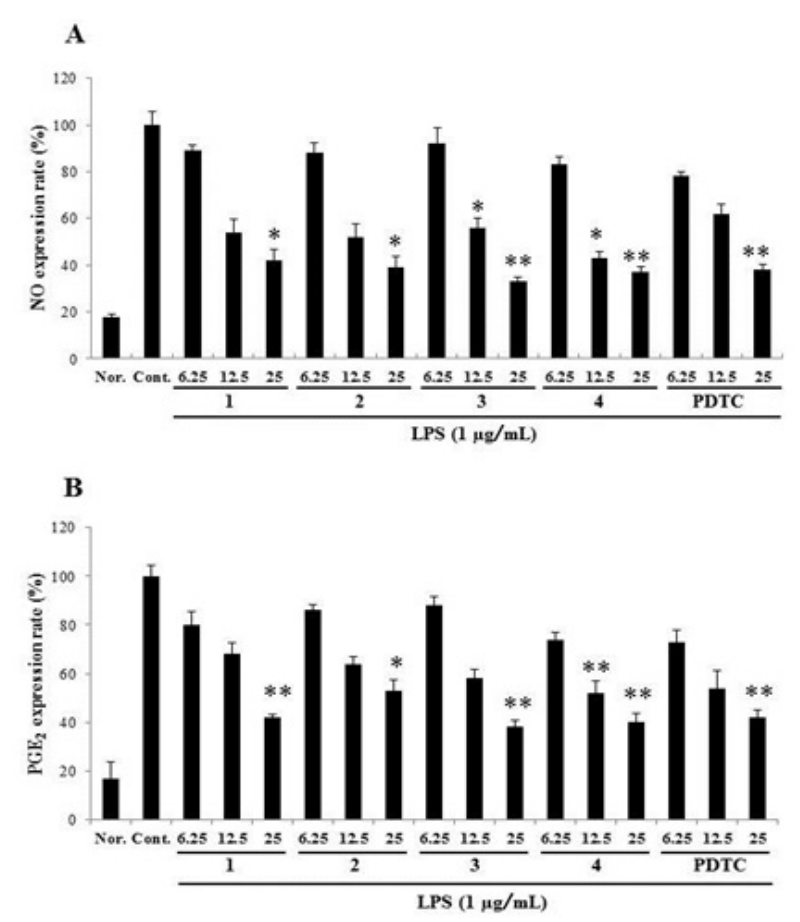

Fig. 2 Effects of four major active compounds of $C$. tinctorius seeds on $\mathrm{NO}(\mathrm{A})$ and $\mathrm{PGE}_{2}$ (B) production by LPS-stimulated RAW 264.7 macrophages. $3 \times 10^{5}$ cells were treated with various concentrations $(6.25,12.5$ and $25 \mu \mathrm{M})$ of each major compound after treatment with $1 \mu \mathrm{g} / \mathrm{mL}$ LPS. PDTC was used as a positive control. 'Nor.' indicates normal RAW cells without any treatment. 'Cont.' represents cells used as negative controls after LPS induction. Values are the mean $\pm \mathrm{SD}$ of three separate experiments. ${ }^{*} p<0.05$, $* * p<0.01$ indicates a significant difference compared to LPS alone
The level of inducible NO synthase (iNOS) and cyclooxygenase-2 (COX-2) was suppressed by serotonins of $C$. tinctorius seeds.

The level of iNOS and COX-2 following treatment with the four major compounds was evaluated by Western blot analysis (Fig. 3 and Fig. 4). The iNOS protein level decreased significantly in LPS-stimulated RAW 264.7 macrophages in response to each tested concentration (Fig. 3). A similar decrease was found in COX-2 protein with $\mathrm{N}$-feruloyl serotonin and $\mathrm{N}$-( $p$-coumaroyl) serotonin inducing the greatest reduction (Fig. 4). These results suggest that phenylpropanoid-conjugated serotonins (Fig. $1 \mathrm{C}$ and $1 \mathrm{D}$ ) and $O$-methylated flavones (Fig. $1 \mathrm{~A}$ and 1B) may defend macrophages from inflammation through suppression of iNOS and COX-2 expression.

Serotonins are found in many plants, as well as in animals and insect venom (Kang et al. 2009). Plant seeds produce serotonins to eliminate accumulated ammonia (Schröder et al. 1999). In addition, serotonins produced by plants may facilitate the removal of seeds from the digestive tract. Moreover, many laxatives are made with seed and fruit extracts containing serotonins. Walnut and hickory have high levels of serotonins (25-400 mg/kg), while pineapples, bananas, kiwis, and tomatoes contain about 3-30 mg/kg (Feldman and Lee 1985). In the present study, C. tinctorius seeds were found to contain 4.3 $\mathrm{mg} / \mathrm{kg}$ serotonin.

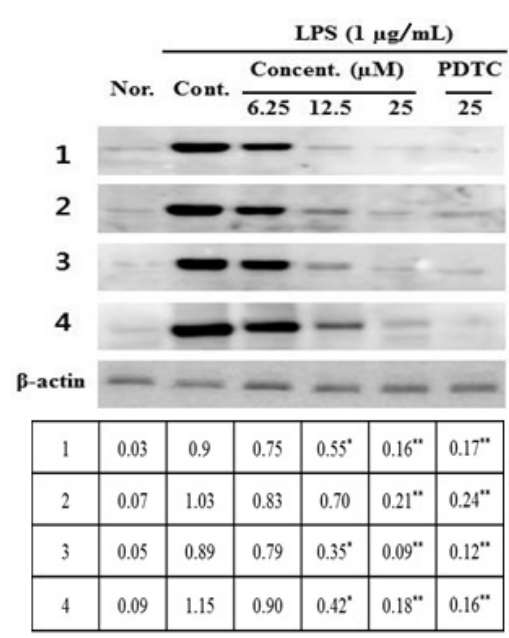

Fig. 3 Changes in iNOS expression level after treatment with four major seed compounds in LPS-stimulated RAW 264.7 macrophages. Cells were treated with compounds after LPS stimulation $(1 \mu \mathrm{g} / \mathrm{mL})$ and the production of iNOS was then quantified by Western blot analysis (upper). Numbers 1-4 indicate four major compounds (1. acacetin, 2. cosmosiin, 3. N-feruloyl serotonin and 4. N-(p-coumaroyl) serotonin). Beta-actin was used as a comparative control. The table shows protein levels normalized by $\beta$-actin using densitometry. ${ }^{*} p$ $<0.05$, ${ }^{* *} p<0.01$ indicates a significant difference compared to the negative control (Cont.) 


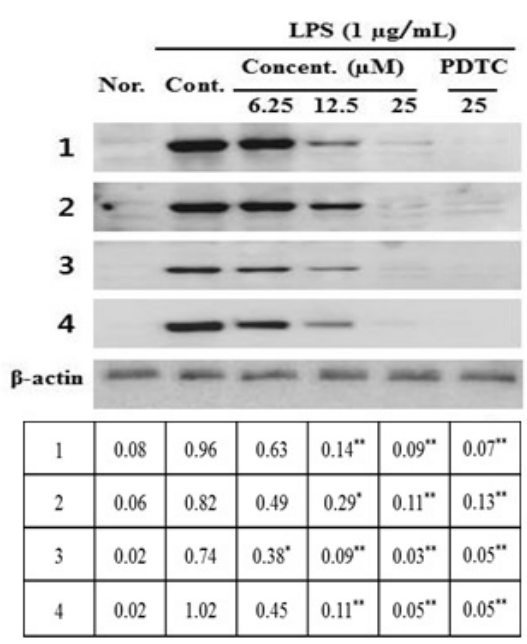

Fig. 4 Effects of four major compounds from C. tinctorius seeds on LPS-stimulated COX-2 expression in RAW 264.7 macrophages. Cells were treated with compounds 1-4 for 1 hour after 24 hours of LPS stimulation $(1 \mu / \mathrm{mL})$. The level of COX-2 was analyzed by Western blot (upper). Numbers 1-4 indicate four major compounds (1. acacetin, 2. cosmosiin, 3. N-feruloyl serotonin and 4. N-( $p$-coumaroyl) serotonin). $\beta$-actin was used as an internal control. The table shows protein levels normalized by $\beta$-actin using densitometry. ${ }^{*} p<0.05$, ${ }^{* *} p<0.01$ indicates a significant difference compared to the negative control (Cont.)

Flowers of safflowers have been used as the traditional remedies of stroke and coronary heart disease in China. However, Korean prefers to use their seeds for the treatments of bone formation, osteoporosis, and prevention of rheumatism. In this study, main active compounds of $C$. tinctorius seeds were identified especially feruloyl serotonins as major chemicals. Serotonins are commonly known for prevention of ammonia accumulation in plants. Apart from that, we found that serotonins isolated from C. tinctorius seeds $(4.3 \mathrm{mg} / \mathrm{kg})$ shows outstanding anti-inflammatory effect in a cell model. In particular, N-feruloyl serotonin and $\mathrm{N}$-(p-coumaroyl) serotonin clearly inhibited inflammatory mediators such as NO and $\mathrm{PGE}_{2}$ via reduction of $\mathrm{NOOS}$ and COX-2 in LPS-stimulated macrophages. It is plausible to say that these major serotonin derivatives from $C$. tinctorius seeds have important antiinflammatory activity and therefore have the potential for pharmaceutical applications.

\section{References}

Abdelrahman M, Sivarajah A, Thiemermann C (2005) Beneficial effects of PPAR-(ligands in ischemia-reperfusion injury, inflammation and shock. Cardiovasc Res 65:772-781

Delgado AV, McManus AT, Chambers JP (2003) Production of tumor necrosis factor-alpha, interleukin 1-beta, interleukin 2, and interleukin 6 by rat leukocyte subpopulations after exposure to substance. Neuropeptides 37:355-361

Feldman JM, Lee EM (1985) Serotonin content of foods: effect on urinary excretion of 5-hydroxyindoleacetic acid. Am J Clin Nutr 42:639-643

Han SY, Li HX, Ma X, Zhang K, Ma ZZ, Tu PF (2009) Protective effects of purified safflower extract on Myocardial ischemia in vivo and in vitro. Phytomedicine 16:694-702

Hewett JA, Roth RA (1993) Hepatic and extra hepatic pathobiology of bacterial lipopolysaccharides. Pharmacol Rev 45:382-411

Kang KY, Park SK, Kim YS, Lee SB, Back KW (2009) Biosynthesis and biotechnological production of serotonin derivatives. Appl Microbiol Biotechnol 83:27-34

Kim DH, Hwang EY, Son JH (2013) Anti-inflammatory activity of Carthamus tinctorious seed extracts in Raw 264.7 cells. J Life science 23:55-62

Kim DH, Moon YS, An BJ, Son JH (2012) Potent anti-aging activity of Aruncus dioicus, a native plant of ulleung-do, south Korea, in CCD-986sk fibroblasts via suppression of matrix metalloproteinases. J Nat Med 66:631-636

Kim IH (1998) Carthamus tinctorius. In: Sinyakboncho. Insandongcheon press, Seoul, Korea, pp. 567-568

Kubes P, McCafferty DM (2000) Nitric oxide and intestinal inflammation. Am J Med 109:150-158

Li HX, Han SY, Wang XW, Ma X, Zhang K, Wang L, Ma ZZ, Tu PF (2009) Effect of the carthamins yellow from Carthamu stinctorius L. on hemorheolo-gical disorders of blood stasis in rats. Food and Chemical Toxicol 47:1797-1802

Mandade R, Sreenivas SA, Wanare R (2012) Antiulcer screening of Carthamus tinctorius on volume and acidity of stimulated gastric secretion in rats. J Pharmacol Pharmacother 3:185-188

Makarov SS (2000) NF- $\kappa$ B as a therapeutic target in chronic inflammation: recent advances. Mol Med Today 6:441-448

Palmer RMJ, Ashton DS, Moncada S (1988) Vascular endothelial cells synthesize nitric oxide from L-arginine. Nature 333:664-666

Roh JS, Han JY, Kim JH, Hwang JK (2004) Inhibitory effects of active compounds isolated from safflower (Carthamus tinctorius L.) seeds for melanogenesis. Biol Pharm Bull 27:1976-1978

Sakamura S, Terayama Y, Kawakatsu S, Ichihara A, Saito H (1978) Conjugated serotonins related to cathartic activity in safflower (Carthamus tinctorious L.) seeds. Agric Biol Chem 42:1805-1806

Sakamura S, Terayama Y, Kawakatsu S, Ichihara A, Saito H (1980) Conjugated serotonins and phenolic constituents in safflower seed (Carthamus tinctorius L.). Agric Biol Chem 44:2951-2954

Seo HJ, Kim JH, Kwak DY, Jeon SM, Ku SK, Lee JH, Moon KD, Choi MS (2000) The effects of safflower seed powder and its fraction on bone tissue in rib-fractured rats during the recovery. Kor J Nutr 33:411-420

Schröder P, Abele C, Gohr P, Stuhlfauth-Roisch U, Grosse W (1999) Latest on enzymology of serotonin biosynthesis in walnut seeds. Adv Exp Med Biol 467:637-644

Surh YJ, Na HK (2008) NF-kB and Nrf2 as prime molecular targets for chemoprevention and cytoprotection with antiinflammatory and antioxidant phytochemicals. Genes Nutr 
2:313-317

Tannenbaum H, Davis P, Russell AS, Atkinson MH, Maksymowych W, Huang SH, Bell M, Hawker GA, Juby A, Vanner S, Sibley J (1996) An evidence-based approach to prescribing NSAIDs in musculoskeletal disease: a Canadian consensus. Canadian NSAID Consensus Participants. Can Med Assoc J 155:77-88
Watson WH, Zhao Y, Chawla RK (1999) S-adenosylmethionine attenuates the lipopolysaccharide-induced expression of the gene for tumor necrosis factor alpha. Biochem J 342:21-25

Xie Q, Nathan C (1994) The high-output nitric oxide pathway: role and regulation. J Leukoc Biol 56:576-592 